TRANSACTIONS OF THE

AMERICAN MATHEMATICAL SOCIETY

Volume 354, Number 6, Pages 2279-2284

S 0002-9947(02)02966-5

Article electronically published on February 14, 2002

\title{
GENERALIZED SPACE FORMS
}

\author{
NEIL N. KATZ AND KEI KONDO
}

\begin{abstract}
Spaces with radially symmetric curvature at base point $p$ are shown to be diffeomorphic to space forms. Furthermore, they are either isometric to $\mathbb{R}^{n}$ or $S^{n}$ under a radially symmetric metric, to $\mathbb{R P}^{n}$ with Riemannian universal covering of $S^{n}$ equipped with a radially symmetric metric, or else have constant curvature outside a metric ball of radius equal to the injectivity radius at $p$.
\end{abstract}

The study of radial sectional curvature on pointed Riemannian manifolds yields generalized Toponogov comparison theorems or generalized Alexandrov convexity theorems in [1], 2], 6] and [9]. Radially symmetric metrics on $\mathbb{R}^{n}$ or $S^{n}$ are used as model spaces taking the place of spaces with constant curvature. Specifically, these model spaces have metrics of the form $d t^{2}+f^{2}(t) d \Theta^{2}$ in radial coordinates where $f(0)=0$ and $f^{\prime}(0)=1$. Additionally, in the case of $\mathbb{R}^{n}, f(t)>0$ for all $t>0$ and for $S^{n}, f(\ell)=0$ and $f^{\prime}(\ell)=-1$ with $f(t)>0$ for $t \in(0, \ell)$. Other results with lower bounds on radial sectional curvature can be found in [10, 11] and references therein.

In this note we show that the more general class of spaces with symmetric radial curvature (defined below) all admit metrics with constant sectional curvature. Furthermore, they are isometric to either a model space (which we call radially symmetric), to $\mathbb{R P}^{n}$ with a radially symmetric $S^{n}$ as a Riemannian cover or have constant sectional curvature outside a metric ball of radius equal to the injectivity radius (Theorem 3 ). In this way the model spaces are a generalization of space forms which are used as models in the original Toponogov comparison theorem. The theorem is a kind of converse to a result of R.E. Greene and H. Wu (7] Proposition 4.2).

The authors would like to thank Katsuhiro Shiohama for suggesting the problem and for helpful discussions. The authors are also grateful to Yukio Otsu and Yukihiro Mashiko for their valuable comments.

Throughout this note $(M, g)$ will denote a smooth, complete, Riemannian manifold of dimension $n \geq 2$ and $\mathrm{d}_{g}(\cdot, \cdot)$ its distance function. Fix a point $p \in M$ and let $\ell:=\sup \left\{\mathrm{d}_{g}(p, q) \mid q \in M\right\}$. A tangent vector $v \neq 0$ is radial to $p$ if there exists a minimal geodesic $\gamma:[0, a] \rightarrow M$ with $\gamma(0)=p$ and $\gamma^{\prime}(a)=v$. We say that $(M, g)$ has symmetric radial curvature $k$ at $p$ if there exists a function $k:[0, \ell) \rightarrow \mathbb{R}$ such that for any tangent vector $v \in T_{q} M$ radial to $p$ and any other vector $w \in T_{q} M$ linearly independent from $v$ the sectional curvature $K_{g}(v, w)=k\left(\mathrm{~d}_{g}(p, q)\right)$.

Received by the editors June 12, 2001 and, in revised form, September 27, 2001.

2000 Mathematics Subject Classification. Primary 53C21; Secondary 53C20.

Key words and phrases. Radial curvature, rigidity.

The first author was supported by the Japan Society for the Promotion of Science and Monbusho Grant-in-Aid of Research No. 13099720. 
For all $v \in S_{p} M:=\left\{u \in T_{p} M \mid\|u\|_{g}=1\right\}$, we will denote by $\rho(v)$ the distance to the cut locus in direction $v$, and the interior set $U:=\left\{t v \in T_{p} M \mid 0 \leq t<\rho(v)\right\}$. The cut locus of $p$ will be denoted by $\operatorname{Cut}(p)$. For a constant $\kappa$, we will write $\operatorname{sn}_{\kappa}(t)=\kappa^{-1 / 2} \sin \sqrt{\kappa} t$ when $\kappa>0, \operatorname{sn}_{0}(t)=t$, and $\operatorname{sn}_{\kappa}=|\kappa|^{-1 / 2} \sinh \sqrt{|\kappa|} t$ for $\kappa<0$. Henceforth we adopt the convention that all geodesics have unit speed unless specifically stated otherwise in which case they will have constant speed.

First we prove some simple facts about metrics with radially symmetric curvature and then prove our main lemma. Finally, we give a proof of the theorem.

Lemma 1. If $\left(M^{n}, g\right)$ has symmetric radial curvature $k:[0, \ell) \rightarrow \mathbb{R}$ at $p$, then the following hold.

(i) The function $k$ is smooth on $[0, \ell)$.

(ii) If $\gamma_{1}, \gamma_{2}:[0, a] \rightarrow M$ are minimal geodesics emanating from $p$ and $Y_{1}, Y_{2}$ are Jacobi fields along $\gamma_{1}, \gamma_{2}$, respectively with $\left\|Y_{1}(0)\right\|_{g}=\left\|Y_{2}(0)\right\|_{g},\left\langle Y_{j}, \gamma_{j}^{\prime}\right\rangle_{g}=$ $0(j=1,2)$, and $\left\|Y_{1}^{\prime}(0)\right\|_{g}=\left\|Y_{2}^{\prime}(0)\right\|_{g}$, then $\left\|Y_{1}(t)\right\|_{g}=\left\|Y_{2}(t)\right\|_{g}$ for all $0 \leq t \leq a$.

(iii) Let $f:[0, \ell) \rightarrow \mathbb{R}$ be the unique solution to the differential equation $f^{\prime \prime}+k f=0$ with initial conditions $f(0)=0$ and $f^{\prime}(0)=1$. Take polar coordinates $(t, \Theta)$ on $\mathbb{R}^{n}$ and define a metric $h=d t^{2}+f^{2}(t) d \Theta^{2}$ on $B(0, T)$ for $t<T=$ $\sup \{r>0 \mid f(t)>0 \forall t \in(0, r)\}$. If we fix an isomorphism $\mathcal{I}: \mathbb{R}^{n} \rightarrow T_{p} M$, then $V=\mathcal{I}^{-1} U \subset B(0, T)$, and $\left.\exp _{p} \circ \mathcal{I}\right|_{V}: V \rightarrow M \backslash \operatorname{Cut}(p)$ is an isometry.

(iv) If $n \geq 3$ then $(V, h)$ defined in (iii) has the property that for any unit vectors $v_{1}, v_{2} \in T_{x} V$ with $\left\langle v_{1}, v_{2}\right\rangle_{h}=0$ and $\left\langle v_{j}, \partial_{t}\right\rangle_{h}=0$, for $j=1,2$ then, $\left\langle R_{h}\left(\partial_{t}, v_{j}\right) \partial_{t}, v_{j}\right\rangle_{h}=k,\left\langle R_{h}\left(v_{1}, v_{2}\right) v_{1}, v_{2}\right\rangle_{h}=\lambda$, and $\left\langle R_{h}\left(v_{1}, v_{2}\right) v_{1}, \partial_{t}\right\rangle_{h}=0$, where $\lambda=\left(1-\left(f^{\prime}\right)^{2}\right) / f^{2}$. Furthermore, the sectional curvature of any plane lies between the two values $k$ and $\lambda$.

Proof. There must exist a minimal geodesic $\gamma:[0, \ell) \rightarrow M$ with $\gamma(0)=p$. Let $E$ be any parallel unit field along $\gamma$ with $\left\langle E, \gamma^{\prime}\right\rangle_{g}=0$. Then $K_{g}\left(E(t), \gamma^{\prime}(t)\right)=k(t)$ for all $0 \leq t<\ell$ and (i) follows.

To show (ii), let $X$ be a parallel unit field along $\gamma_{1}$ with $\left\langle X, \gamma_{1}^{\prime}\right\rangle_{g}=0$. If $n \geq 3$ we have that for any parallel unit field $Y$ along $\gamma_{1}$ such that $\left\{X, Y, \gamma^{\prime}\right\}$ are orthonormal, $\left\langle R_{g}\left(\gamma_{1}^{\prime}, X+Y\right) \gamma_{1}^{\prime}, X+Y\right\rangle_{g}=2 K\left(X+Y, \gamma_{1}^{\prime}\right)=2 k(t)$, hence, $\left\langle R_{g}\left(\gamma_{1}^{\prime}, X\right) \gamma_{1}^{\prime}, Y\right\rangle_{g}=0$. Therefore $R_{g}\left(\gamma_{1}^{\prime}, X\right) \gamma_{1}^{\prime}=k X$. Similarly, the same relation holds along $\gamma_{2}$. It is easy to show this holds also when $n=2$ and (ii) follows quickly.

Pick $x \in \mathcal{I}^{-1} U$ and let $v=\mathcal{I} x$ and $q=\exp _{p} v$. Set $\gamma_{v}(t)=\exp _{p}(t v)$ and fix a parallel unit frame field $\left\{E_{1}, \ldots, E_{n}\right\}$ along $\gamma_{v}$ with $E_{1}$ tangent to $\gamma_{v}$. Take $\beta$ to be a minimal $h$-geodesic from the origin to $x$. Then $R_{h}\left(\beta^{\prime}, Z\right) \beta^{\prime}=k Z$ for any perpendicular field $Z$ along $\beta$. Whence if $\left\{\bar{E}_{1}, \ldots, \bar{E}_{n}\right\}$ is a parallel frame field along $\beta, f \bar{E}_{j}$ is a Jacobi field for any $j$. We have that for any $v \in U,\left(d \exp _{p}\right)_{v}(w)=Y(1)$, where $Y$ is the unique Jacobi field along the geodesic $\gamma_{v}(t)=\exp _{p}(t v)$ such that $Y(0)=0$ and $Y^{\prime}(0)=w$. Thus, the Jacobi fields along geodesics emanating from $p$ satisfy the same equation as those emanating from $0 \in(B(0, T), h)$. It follows from a well known argument that $\left.\exp _{p} \circ \mathcal{I}\right|_{V}$ is an isometry.

Finally, (iv) follows from standard calculations using the Gauss and Codazzi equations and the fact that $f^{\prime \prime}+k f=0$.

The metrics defined in Lemma 1(iii) above give complete manifolds either when $M$ is non-compact and $f>0$, in which case $M$ is diffeomorphic to $\mathbb{R}^{n}$, or else if 
$M$ is compact and $f^{\prime}(r)=-1$ where $f(r)=0$ is the first zero, in which case $M$ is diffeomorphic to $S^{n}$.

Lemma 2. Let $(M, g)$ be a complete $n$-manifold with symmetric radial curvature $k:[0, \ell) \rightarrow \mathbb{R}$ at $p$ such that $a:=\operatorname{inj}(p)<\ell$. Then there are no points conjugate to $p$ at a distance less than $\ell$. Furthermore, for any $q \in \operatorname{Cut}(p)$ if $b:=\mathrm{d}_{g}(p, q) \in(a, \ell)$, then $k^{\prime}(b)=0$.

Proof. From Lemma 1(ii) it follows that there are no points with distance to $p$ greater than the distance to the first conjugate point and the first claim follows as $\ell=\sup \left\{\mathrm{d}_{g}(p, q) \mid q \in M\right\}$.

The proof of the second claim will be by contradiction. Suppose that $k^{\prime}(b) \neq 0$. Then for some $\delta_{0}>0$ we have $\left|k^{\prime}(t)\right|>0$ for all $t \in\left(b-2 \delta_{0}, b+2 \delta_{0}\right)$. We will choose a geodesic $\gamma:[0, \ell) \rightarrow M$ emanating from $p$, minimal on $[0, b]$ with $\gamma(b)=q$, and construct a vector field $Y$ along $\left.\gamma\right|_{[b-\delta, b+\delta]}$ for some $0<\delta \leq \delta_{0}$ as follows. If one exists, we take $\gamma$ to be a geodesic loop at $p$ with $\left.\gamma\right|_{[0, b]}$ and $\left.\gamma\right|_{[b, 2 b]}$ distinct, minimal geodesics between $p$ and $q$. Thus $\gamma^{\prime}(t)$ is radial for $t \in\left(b-\delta_{0}, b\right]$, and $-\gamma^{\prime}(t)$ is radial for $t \in\left[b, b+\delta_{0}\right)$. Let $Y$ be a parallel unit field with $\left\langle\gamma^{\prime}(t), Y(t)\right\rangle_{g}=0$ and take $\delta=\delta_{0}$.

Otherwise, if there are no geodesic loops at $p$ through $q$, fix a geodesic $\gamma: \mathbb{R} \rightarrow$ $M$ with $\gamma(0)=p$ and $\gamma(b)=q$. There exists $\epsilon>0$ such that $\exp _{p}^{-1} B_{\epsilon}(q)$ is a disjoint union of finitely many connected components $\mathcal{O}_{1}, \ldots, \mathcal{O}_{m}$. Take $0<$ $\delta_{1}<\delta_{0}$ such that $\left.\gamma\right|_{\left[b, b+\delta_{1}\right)} \subset B_{\epsilon}(q)$ and let $\xi_{1}, \ldots, \xi_{m}:\left[b, b+\delta_{1}\right) \rightarrow T_{p} M$ be its lifts. Since $\left.\exp _{p}\right|_{\mathcal{O}_{j}}$ is a diffeomorphism onto its image, $\xi_{j}$ is smooth for all $j=1, \ldots, m$. By continuity there exists $j_{0}$ and $0<\delta<\delta_{1}$ such that $\left\|\xi_{j_{0}}(t)\right\|_{g}=$ $\min \left\{\left\|\xi_{1}(t)\right\|_{g}, \ldots,\left\|\xi_{m}(t)\right\|_{g}\right\}$ for all $t \in[0, \delta)$. Let $\beta_{t}(s)=\exp _{p}\left(s \xi_{j_{0}}(t)\right)$, and $Y(t)=$ $\beta_{t}^{\prime}(1)$ for $b \leq t<b+\delta$. Extend $Y$ smoothly to $(b-\delta, b]$ so that $\left\{Y, \gamma^{\prime}\right\}$ are linearly independent. We have that $\gamma^{\prime}(t)$ is radial for $t \in(b-\delta, b]$, and $Y(t)$ is radial for $t \in[b, b+\delta)$.

Let $\sigma(t)=\operatorname{span}\left\{Y(t), \gamma^{\prime}(t)\right\}$ and consider the function $\eta(t)=K_{g}(\sigma(t))$. It is smooth on $(b-\delta, b+\delta)$. We have for $t \in(b-\delta, b+\delta)$,

$$
\eta(t)=K_{g}(\sigma(t))=k\left(\mathrm{~d}_{g}(p, \gamma(t))\right)
$$

since by construction $\sigma$ always contains a vector radial to $p$. As $\gamma$ is minimizing on $[0, b]$, for all $t \in[0, b]$,

$$
\eta(t)=K_{g}(\sigma(t))=k(t)
$$

Now $q$ is not conjugate to $p$ along any geodesic of length less than $\ell$. Hence there exists $-1 \leq \theta_{0}<1$ such that for any minimal geodesic $\bar{\gamma}$ from $p$ to $q,\left\langle\bar{\gamma}^{\prime}(b), \gamma^{\prime}(b)\right\rangle_{g}<$ $\theta_{0}$. The first variation formula gives that if $\theta_{0}<\theta<1$ is fixed then for all $t>b$ sufficiently small, $\mathrm{d}_{g}(p, \gamma(t))<b+\theta(t-b)$. Since (11) holds and $\left|k^{\prime}\right|>0$ on $(b-\delta, b+\delta)$, therefore the derivative from the right hand side, $\left|\eta^{\prime}\left(b^{+}\right)\right|<\theta\left|k^{\prime}(b)\right|$. This and (2) together contradict the smoothness of $\eta$, proving the lemma.

Theorem 3. Let $(M, g)$ be a complete $n$-dimensional Riemannian manifold with symmetric radial curvature $k:[0, \ell) \rightarrow \mathbb{R}$ at $p$. Then one of the following (nonexclusive) possibilities hold:

(i) The sectional curvature $K_{g}$ is constant outside $B_{a}(p)$ where $a=\operatorname{inj}(p)$ in which case $M$ admits a metric with constant sectional curvature (which is non-positive in the case that $M$ is not compact). Furthermore, $\left(B_{a}(p), g\right)$ is isometric to a radially symmetric metric on an open ball in $\mathbb{R}^{n}$. 
(ii) $(M, g)$ is isometric to $\mathbb{R}^{n}$ with a radially symmetric metric.

(iii) $(M, g)$ is isometric to $S^{n}$ with a radially symmetric metric.

(iv) $(M, g)$ is diffeomorphic to $\mathbb{R P}^{n}$ and its universal Riemannian covering is isometric to $S^{n}$ with a radially symmetric metric.

Proof. Define $V \subset B(0, T) \subset \mathbb{R}^{n}$, the map $\varphi: V \rightarrow M$, and the metric $h=d t^{2}+$ $f^{2}(t) d \Theta^{2}$ in polar coordinates on $B(0, T)$ as in Lemma 1(iii). From Lemma प(iii), $\varphi$ is an isometry. From Lemma[1(ii) the first conjugate locus of $p$ does not intersect $B_{\ell}(p)$. Therefore $f(t)>0$ for all $0<t<\ell$.

We will first consider the case that $a=\operatorname{inj}(p)<\ell \leq \infty$. From Lemma [2] $k^{\prime}(r)=0$ for all $a \leq r<\ell$. So $k(t) \equiv \kappa$ for $t \in[a, \ell)$. In the case $n=2$ this means that the curvature outside of $B_{a}(p)$ is constant. Now assume that $n \geq 3$. Take $q \in \operatorname{Cut}(p)$ with $\mathrm{d}_{g}(p, q)=r \in(a, \ell)$. By Lemma $1\left(\right.$ iv), if $u \in T_{q} M$ is any radial vector and $x, y \in T_{q} M$ are such that $\{u, x, y\}$ is orthonormal, then $K_{g}(u, x)=\kappa$, and $K_{g}(x, y)=\lambda\left(\mathrm{d}_{g}(p, q)\right)$ (with notation as in Lemma 1). The theory of critical points for distance functions (see the surveys [5] or 8 for definitions and proofs) gives that if $q$ is a cut point but not a critical point of the distance function to $p$, then there exist distinct, radial, unit vectors $u, v \in T_{q} M$ with $\mathrm{d}_{g}(p, q)=r$ and $\langle u, v\rangle_{g}=$ $\alpha \in(-1,1)$. Let $u^{\prime}=u-\alpha v$, and $v^{\prime}=v-\alpha u$. Thus $\left\langle u^{\prime}, v\right\rangle_{g}=\left\langle v^{\prime}, u\right\rangle_{g}=0$. For any unit vector $w$ with $\langle w, u\rangle_{g}=\langle w, v\rangle_{g}=0, R\left(u^{\prime}, w, u^{\prime}, w\right)=\left\|u^{\prime}\right\|_{g}^{2} K_{g}\left(u^{\prime}, w\right)=$ $\left(1-\alpha^{2}\right) \lambda(r)$. On the other hand, $R\left(u^{\prime}, w, u^{\prime}, w\right)=R(u-\alpha v, w, u-\alpha v, w)=$ $\left(1-\alpha^{2}\right) \kappa-2 \alpha R\left(w, v^{\prime}, w, u\right)$. Applying Lemma 1(iv) we have that $R\left(w, v^{\prime}, w, u\right)=0$ and so $\left(1-\alpha^{2}\right)(\kappa-\lambda(r))=0$, hence $\lambda(r)=\kappa$. Therefore all sectional curvatures at $q$ are equal to $\kappa$. Since regular points are a dense subset it follows that $K_{g}(\sigma)=\kappa$ for all two dimensional subspaces $\sigma \subset T_{q} M$ and all $q \in M \backslash B_{a}(p)$. Furthermore, if $M$ is non-compact, the constant $\kappa \leq 0$. Since $\exp _{p}(U)$ is an open dense subset of $M$ and is isometric to $(V, h)$, it follows that $(M, g)$ has constant sectional curvature outside $B_{a}(p)$. Let $q$ be a cut point to $p$ realizing $\mathrm{d}_{g}(p, q)=\operatorname{inj}(p)$. Since $a=\operatorname{inj}(p)<l, q$ is not conjugate to $p$ so there is a geodesic loop $\gamma:[0,2 a] \rightarrow M$ with $\gamma(0)=\gamma(2 a)=p$ and $\gamma(a)=q$ such that $\left.\gamma\right|_{[0, a]}$ and $\left.\gamma\right|_{[a, 2 a]}$ are minimal. Let $Y$ be a parallel unit vector field along $\gamma$ with $g\left(\gamma^{\prime}, Y\right) \equiv 0$. Define a vector field $J(t)=F(t) Y(t)$ with $F(t)=f(t)$ for $t \in[0, a]$ and $F(t)=f(2 a-t)$ for $t \in[a, 2 a]$. By Lemma 1(ii),(iii) $J$ is a once broken Jacobi field. Since $q$ realizes the distance between $p$ and the cut locus of $p$, the second variation of the energy of $\gamma$ is non-negative. The second variation formula then gives that $f(a) f^{\prime}(a) \geq 0$. Since $f(a)>0$ we conclude that $f^{\prime}(a) \geq 0$. Now $f^{\prime \prime}+\kappa f=0$ on $[a, \ell)$, and by Lemma[1(iv), $1-\left(f^{\prime}\right)^{2}=f^{2} \kappa$ on $(a, \ell)$. It follows that $f(t)=\operatorname{sn}_{\kappa}(t+b)$ for $t \in[a, \ell)$ where $b$ (the constant of integration) is such that $\operatorname{sn}_{\kappa}(a+b)=f(a)>0$. We have that $\exp _{p}: B(0, \ell) \rightarrow M$ is an immersion, whence it is easy to construct a smooth metric $\tilde{g}$ on $M$ such that $\tilde{g}=g$ on $M \backslash B_{a}(p)$ and $\tilde{g}$ has constant sectional curvature $\kappa$ on $B_{a}(p)$. Thus $\tilde{g}$ has constant sectional curvature everywhere and we have shown that (i) holds.

In the case that $M$ is non-compact and $p$ has no cut points, that is $\operatorname{inj}(p)=\ell=$ $\infty$, the first conjugate locus of $p$ is empty so $f(t)>0$ for all $t>0$. Since $\varphi$ is an isometry and $\operatorname{inj}(p)=\infty$, (ii) follows.

Now we turn to the case that $M$ is compact and $\operatorname{inj}(p)=\ell$. Let $q \in \operatorname{Cut}(p)$ realize $a=\mathrm{d}_{g}(p, \operatorname{Cut}(p))$. If $q$ is conjugate to $p$ along a geodesic of length $a$ then applying Lemma 1(ii), the multiplicity must be maximal and indeed any geodesic emanating from $p$ is minimizing up to length $a$ where it has a conjugate point of maximal multiplicity. In this case a theorem due to A. Allamigeon [3] 
(Sections 3 and 4) and F.W. Warner [12] (see also 44 5.29), gives that $(M, g$ ) is homeomorphic to $S^{n}$. Next we are going to show that $(M, g)$ is isometric to $S^{n}$ with a radially symmetric metric. Define a diffeomorphism $\Phi: \mathbf{S}_{p}^{n-1} \rightarrow \mathbf{S}_{q}^{n-1}$, where $\mathbf{S}_{p}^{n-1}$ and $\mathbf{S}_{q}^{n-1}$ are the unit hyperspheres centered at the origins of $T_{p} M$ and $T_{q} M$, respectively as follows. Let $\Phi\left(\tau^{\prime}(0)\right)=-\tau^{\prime}(a)$, where $\tau:[0, a] \rightarrow M$ is a minimal geodesic from $p$ to $q$. Since $\operatorname{Cut}(p)=\{q\}$ the map is well-defined. For $\tau^{\prime}(0) \in \mathbf{S}_{p}^{n-1}$ we choose a local coordinate $\left(\theta^{1}, \cdots, \theta^{n-1}\right)$ on a domain in $\mathbf{S}_{p}^{n-1}$ containing $\tau^{\prime}(0)$ such that $\left\langle\partial_{\theta^{i}}\left(\tau^{\prime}(0)\right), \partial_{\theta^{j}}\left(\tau^{\prime}(0)\right)\right\rangle_{\left(\mathbf{S}_{p}^{n-1} \text {, can }\right)}=\delta_{i j}$. Applying Lemma 1(ii), for $i=1, \ldots, n-1, Y_{i}(t)=f(t) e_{i}(t)$ are the Jacobi fields along $\tau$, for $t \in[0, a]$, where $\left\{e_{1}, \ldots, e_{n-1}\right\}$ are parallel orthonormal fields along $\tau$ with $\left\langle e_{i}(t), \tau^{\prime}(t)\right\rangle_{g}=0$ such that $e_{i}(0)=\partial_{\theta^{i}}\left(\tau^{\prime}(0)\right)$. Since $d \Phi: T \mathbf{S}_{p}^{n-1} \rightarrow T \mathbf{S}_{q}^{n-1}$ is given by $d \Phi_{\tau^{\prime}(0)}\left(Y_{i}^{\prime}(0)\right)=-Y_{i}^{\prime}(a)=-f^{\prime}(a) e_{i}(a)(i=1, \cdots, n-1)$, we see that $\operatorname{Vol}\left(\mathbf{S}_{q}^{n-1}\right)=\int_{\mathbf{S}_{p}^{n-1}} \Phi^{*} \mu=\left|f^{\prime}(a)\right|^{n-1} \operatorname{Vol}\left(\mathbf{S}_{p}^{n-1}\right)$, where $\mu$ is the volume element on $\mathbf{S}_{q}^{n-1}$. This implies $\left|f^{\prime}(a)\right|=1$ so $f^{\prime}(a)=-1$ since $q$ is conjugate to $p$ along $\tau$ and $f^{\prime}(0)=1$. Therefore $M$ has the metric $g=d t^{2}+f^{2}(t) d \Theta^{2}$ with $f(0)=f(a)=0, f^{\prime}(0)=1$, and $f^{\prime}(a)=-1$, which implies (iii).

The remaining case is when $\operatorname{inj}(p)=\ell<\infty$ and there are no points conjugate to $p$ along any geodesic of length $\ell$. It follows that every geodesic emanating from $p$ is a loop of length $2 \ell$ minimizing up to length $\ell$. Therefore $p$ is conjugate to itself at distance $2 \ell$ along any of these geodesic loops and Lemma 1(ii) gives that the multiplicity is $n-1$. Call one of these loops $\gamma$ and for any parallel unit vector field $Y$ along it with $\left\langle Y, \gamma^{\prime}\right\rangle_{g}=0$, let $\bar{k}(t)=K_{g}\left(Y(t), \gamma^{\prime}(t)\right)$ for $t \in[0,2 \ell]$. Since $\mathrm{d}_{g}(\gamma(t), p)=t$ for $t \in[0, \ell]$ and $\mathrm{d}_{g}(\gamma(t), p)=2 \ell-t$ for $t \in[\ell, 2 \ell]$, we have that $\bar{k}(2 l-t)=\bar{k}(t)$ for all $t \in[0,2 \ell]$. As $p$ is conjugate to itself along $\gamma$, there exists a solution to $y^{\prime \prime}+\bar{k} y=0$ with $y(0)=y(2 \ell)=0$ and $y^{\prime}(0)=1$. Let $\bar{y}(t)=y(2 \ell-t)$. Then the symmetry of $\bar{k}$ about $\ell$ gives that $\bar{y}^{\prime \prime}(t)+\bar{k}(t) \bar{y}(t)=0$. We have that $y(t)>0$ for all $t \in(0,2 \ell)$ as follows. We know this holds for $t \in(0, \ell]$ as $p$ has no conjugate points before distance $\ell$. Suppose that $y\left(t_{0}\right)=0$ for some $t_{0} \in(\ell, 2 \ell)$. Then $\bar{y}\left(2 \ell-t_{0}\right)=y\left(t_{0}\right)=0=\bar{y}(0)$, a contradiction. It follows that $y^{\prime}(2 \ell)=-a^{2} \leq 0$ and so $z=(y+\bar{y}) /\left(1+a^{2}\right)$ is also a solution of the differential equation with the same initial conditions as $y$, thus $z=y$, so $\bar{y}^{\prime}(2 \ell)=a^{2} y^{\prime}(2 \ell)$. Hence $\bar{y}=y$ and in particular $-a^{2}=y^{\prime}(2 \ell)=-1$. By a now familiar argument we construct a radially symmetric metric on $S^{n}$, by letting $\bar{g}=d t^{2}+y^{2}(t) d \Theta^{2}$ in polar coordinates at a point $\bar{p}$. Note that $\left(S^{n}, \bar{g}\right)$ has diameter $2 \ell$ realized by all geodesics emanating from $p$. This manifold gives a double cover of $(M, g)$ by identifying the unit tangent spheres at $\bar{p}$ and $p$ with an isometry and mapping geodesics emanating

from $p$ of length $2 \ell$ to geodesic loops at $p$ of length $2 \ell$. We conclude that $(M, g)$ is diffeomorphic to $\mathbb{R P}^{n}$ and (iv) follows.

\section{REFERENCES}

[1] U. Abresch, Lower curvature bounds, Toponogov's theorem and bounded topology I, Ann. Sci. Ecole Norm. Sup., 19 (1985) 651-670. MR 87j:53058

[2] U. Abresch, Lower curvature bounds, Toponogov's theorem and bounded topology II, Ann. Sci. Ecole Norm. Sup., 20 (1987) 475-502. MR 89d:53080

[3] A. Allamigeon, Propriétés globales des espaces de Riemann harmoniques, Ann. Inst. Fourier, 15 (1965) 91-132. MR 33:6549

[4] A.L. Besse, Manifolds all of whose Geodesics are Closed, Springer-Verlag, Berlin-Heidelberg, 1978. MR 80c:53044 
[5] J. Cheeger, Critical Points of Distance Functions and Applications to Geometry, in Geometric Topology: Recent Developments, Lecture Notes in Math. 1504, Springer-Verlag, Berlin-Heidelberg, 1991. MR 94a:53075

[6] D. Elerath, An improved Toponogov comparison theorem for non-negatively curved manifolds, J. Differential Geometry, 15 (1980) 187-216. MR 83b:53039

[7] R.E. Greene and H. Wu, Function Theory on Manifolds which Possess a Pole, Lecture Notes in Math. 699, Springer-Verlag, Berlin-Heidelberg, 1979. MR 81a:53002

[8] K. Grove, Critical Point Theory for Distance Functions, Proc. of Symposia in Pure Math., 54 Part 3, Amer. Math. Soc., Providence, RI, 1993. MR 94f:53065

[9] Y. Itokawa, Y. Machigashira and K. Shiohama, Generalized Toponogov's theorem for manifolds with radial curvature bounded below, preprint.

[10] Y. Machigashira and K. Shiohama, Riemannian manifolds with positive radial curvature, Japan. J. Math., 19 (1994) 419-430. MR 95f:53080

[11] V. Marenich, Manifolds with minimal radial curvature bounded from below and big volume, Trans. Amer. Math. Soc., 352 (2000) 4451-4468.

[12] F.W. Warner, Conjugate loci of constant order, Ann. of Math., 86 (1967) 192-212. MR 35:4857

Department of Mathematics, Faculty of Science and Engineering, Saga University, HONJOH 1, SAGA 840-8502, JAPAN

E-mail address: katz@ms.saga-u.ac.jp

Department of Mathematics, Faculty of Science and Engineering, Saga University, HONJOH 1, SAGA 840-8502, JAPAN

E-mail address: kondok@ms.saga-u.ac.jp 